\title{
Trophic And Immunomodulatory Effects of Adipose Tissue-Derived Stem Cells In A Preclinical Murine Model of Endometriosis
}

\section{Toyofumi Hirakawa}

Fukuoka University

Fusanori Yotsumoto

Fukuoka University

Naoto Shirasu

Fukuoka University

Chihiro Kiyoshima

Fukuoka University

Daichi Urushiyama

Fukuoka University

Kenichi Yoshikawa

Fukuoka University

Kohei Miyata

Fukuoka University

Masamitsu Kurakazu

Fukuoka University

Kaori Azuma Koga

Fukuoka University

Mikiko Aoki

Fukuoka University

Kazuki Nabeshima

Fukuoka University

Yutaka Osuga

Tokyo University

Hiroaki Komatsu

Tottori University

Fuminori Taniguchi

Tottori University

Tasuku Harada

Tottori University

Shin'ichiro Yasunaga 
Fukuoka University

Shingo Miyamoto ( $\square$ smiya@cis.fukuoka-u.ac.jp )

Fukuoka University

Kaori S Koga

Tokyo University

\section{Research Article}

Keywords: Endometriosis, pathological, fibrosis, endometriosis

Posted Date: September 2nd, 2021

DOl: https://doi.org/10.21203/rs.3.rs-855777/v1

License: (c) (1) This work is licensed under a Creative Commons Attribution 4.0 International License. Read Full License 


\section{Abstract}

Endometriosis, which exhibits enigmatic pathological features such as stromal fibrosis and proliferation of ectopic epithelial cells, is known as a refractory disease. Mesenchymal stem cells modulate the fibrosis in stromal tissues through their trophic and immunomodulatory properties. To investigate the potential of stem cells in treating endometriosis, we examined the secondary morphology and molecular alterations in endometriosis-like lesions after the administration of adipose tissue-derived stem cells (ASCs) to an experimental murine model of endometriosis. The infused ASCs were found integrated in the endometriosis-like lesions. Accompanied by the suppression of stromal fibrosis and proliferation of endometriotic epithelial cells, the infusion of ASCs with stemness potential suppressed the growth of endometriosis-like lesions and inhibited the expression of pro-inflammatory and pro-fibrotic cytokines, whereas no significant attenuation of endometriosis-like lesions occurred after the infusion of ASCs without stemness potential. Accordingly, it is plausible that the trophic and immunomodulatory properties of ASCs may regulate fibrosis in endometriosis-like lesions, suggesting that regenerative medicine has strong potential as an innovative treatment for patients with endometriosis.

\section{Introduction}

Endometriosis, a clinically common inflammatory gynecologic disorder, involves the growth of endometrial-like glands and stroma harboring reactive fibrosis and metaplasia outside the uterus [1]. It is estimated to affect at least $6-10 \%$ of women with reproductive potential [2] and is characterized by pelvic pain, dysmenorrhea, dyspareunia, infertility, dysuria, and dyschezia [3]. Moreover, ovarian endometriosis is found in $20-55 \%$ of women with endometriosis [4], and accumulating evidence has demonstrated an association between ovarian endometriosis and ovarian cancer [5]. It has also been reported that malignant transformation occurs in up to $1 \%$ of patients with ovarian lesions [6]. Accordingly, women of reproductive age are prone to disorders associated with endometriosis. To the best of our knowledge, endometriosis has been commonly treated by excising peritoneal lesions and ovarian cysts or administrating hormonal agents including progestin, oral contraceptives, and gonadotropin-releasing hormone agonists or antagonists. Such treatment strategies have been associated with high recurrence rates [7]; therefore, there is a need for more effective therapies for endometriosis.

The pathophysiology of endometriosis, which is characterized by fibrosis, resistance to apoptosis, and promotion of cell proliferation, has not yet been fully elucidated. Fibrosis is consistently found in all forms of endometriosis. Platelets, macrophages, ectopic endometrial cells, and sensory nerve fibers are involved in the development of fibrosis in all endometrial lesions [8]. Chronic inflammatory conditions have been linked to a variety of fibrotic disorders, including retroperitoneal fibrosis, hepatic fibrosis, idiopathic pulmonary fibrosis, systemic sclerosis (CREST syndrome), Crohn's disease, radiation enteritis, collagenous colitis, encapsulating peritoneal sclerosis, and endometriosis [9]. It has been thought that persistently high levels of TGF- $\beta 1$ or stimulation by inflammatory cytokines (e.g., IL-6) induce adhesion and fibrosis in these diseases $[10,11]$. Thus, chronic exposure to pro-inflammatory and pro-fibrotic 
cytokines may inflict damage on indigenous MSC-like cells by triggering epigenetic modifications and activating matrix-related genes, resulting in myofibroblast differentiation and fibrosis [12]. According to these lines of evidence, it is plausible that the abnormal activation of TGF- $\beta 1$ and upregulation of proinflammatory cytokines result in the development of fibrosis in endometriotic lesions $[13,14]$.

Stem cells possess self-renewal ability and can differentiate into a variety of specialized cell types, retaining infinite regenerative potential through trophic and immunomodulatory properties [15-19]. There are four main sources of stem cells: embryonic tissues, fetal tissues, adult tissues, and differentiated somatic cells [20]. In addition, genetically reprogrammed cells, referred to as induced pluripotent stem cells (iPSCs), can be obtained after the transfection of specific genes [20]. Regenerative therapy is defined as the therapeutic application of stem cells, progenitor cells, or both to repair damaged organs and restore their functions [21-24]. Thus far, embryonic stem cell- or iPSC-based therapies have not been successfully translated into clinical practice for disease treatment [25]. Meanwhile, mesenchymal stem cells (MSCs) have been recognized as stromal cells harboring no regenerative properties but with trophic and immunomodulatory properties, including angiogenesis and immune modulation [25]. Regenerative therapies using such stem cells have been adapted in preclinical and clinical trials [21-24]. In principle, MCSs exert direct and indirect trophic as well as immunomodulatory properties to induce regenerative processes in damaged tissues [26]. MSCs directly exert trophic effects through the secretion of numerous paracrine factors via the secretosome and extracellular vesicles [26]. For its indirect effects, MSCs regulate the expression of pro-inflammatory and anti-inflammatory cytokines through the activity of $T$ and B lymphocytes [26]. Thus, MSCs have been shown to be useful in treating a variety of chronic inflammatory disorders [27-33].

To investigate the therapeutic potential of MSCs for the treatment of endometriosis, we generated an experimental murine model of endometriosis and subjected it to adipose tissue-derived stem cell (ASC) infusion. We then examined alterations in endometriosis-like lesions and assessed the accumulation of labeled ASCs in these lesions after treatment. In addition, we evaluated the gene expression of proinflammatory, anti-inflammatory, and pro-fibrotic cytokines as well as hormone receptors in the endometriosis-like lesions. Ki67 expression was also assessed to determine the proliferative index of endometriotic epithelial cells in endometriosis-like lesions after ASC treatment.

\section{Results}

\section{Cellular characteristics and therapeutic effects of ASCs}

To examine the expression of cell-surface markers of MSCs, flow cytometry was performed using antibodies against CD105, CD29, and Sca-1 as positive markers, and against CD45 as the negative marker. For cells in the P1, P2, and P3 plots, phenotype was analyzed based on stained cell-surface markers (Fig. 1A and Supplementary Fig. S2). The percentage of MSCs in ASCs was $44.8 \pm 3.2 \%(n=3)$ at cell passage 3 (P3) (Fig. 1A), which decreased to $1.7 \pm 0.3 \%(n=3)$ by P20 (Fig. 1A). These results suggest that serial subcultivation induces a marked decrease in the stemness properties of ASCs. 
To investigate the anti-tumor effects of ASCs in the experimental models of endometriosis, we first assessed the total weight, number, and surface area of endometriosis-like lesions after the intravenous administration of phosphate buffer saline (PBS) as control ( $n=10$; Control-Day 28 (D28)), P3 ASCs ( $n=$ 10; 3rd ASCs-P3-D1), and P20 ASCs ( $n=10$; ASCs-P20-D1) to the experimental mice on day 1 ; the mice were then sacrificed on day 28. Next, to evaluate the anti-tumor effects of ASCs on the endometriosis-like lesions that had already formed, we estimated the total weight, number, and surface area of endometriosis-like lesions in control mice $(n=10)$ that were sacrificed on day 15 (Control-D15) as well as mice intravenously administered P3 ASCs ( $n=10$; ASCs-P3-D15) and P20 ASCs ( $=10$; ASCs-P20-D15) on day 15 and sacrificed on day 28.

Endometriosis-like lesions grew in the abdominal cavities of all mice. These lesions were observed on the surface of the peritoneum, liver, kidney, intestinal membrane, and Douglas cavity. After the intravenous administration of ASCs on day 1 and day 15, the total weight, number, and surface area of the endometriosis-like lesions in ASCs-P3 groups were significantly lower than those in the Control-D28 and ASCs-P20 groups (Fig. 1B). These results indicate that the administration of ASCs with stemness potential suppresses the growth of endometriosis-like lesions. In addition, no significant differences in the total weight, number, or surface area of endometriosis-like lesions were found between the ASCs-P3-D1 and ASCs-P3-D15 groups, between the Control-D15 and ASCs-P3-D15 groups, and among the ControlD28, ASC-P20-D1, and ASCs-P20-D15 groups (Fig. 1B). These results indicate that the administration of ASCs with stemness potential inhibits the progression of endometriosis-like lesions.

\section{Macroscopic and microscopic alterations in endometriosis- like lesions mediated by the administration of ASCs}

To evaluate the morphological effects of ASCs on endometriosis-like lesions, we examined the alterations in stromal tissues and endometriotic epithelial cells. Figure 2A shows a representative example of an excised implant from the Control-D28, ASCs-P3-D1, Control-D15, and ASCs-P3-D15 groups. The endometriosis-like lesions formed simple cysts containing a clear fluid, and their diameter ranged from 1 $\mathrm{mm}$ to $10 \mathrm{~mm}$. For evaluating stromal fibrosis, we measured the maximum stromal thickness from the bottom of the endometrium to the surface of each cyst after hematoxylin and eosin staining (Fig. 2B); most stromal tissues consisted of fibroblasts (stained in red) and collagen fibers (stained blue). The stromal tissues in endometriosis-like lesions were comprised primarily of collagen fibers (Fig. 2C). The maximum stromal thickness in the ASCs-P3-D1, Control-D15, and ASCs-P3-D15 groups was significantly lower than that in the Control-D28 group (Fig. 2B and Fig. 2E). However, no significant difference in maximum stromal thickness or stromal fibrosis was detected among the ASCs-P3-D1, Control-D15, and ASCs-P3-D15 groups (Fig. 2B, Fig. 2C, and Fig. 2E). These results suggest that endometriosis-like lesions gradually grow concurrent with stromal fibrosis and that infusion of ASCs with stemness potential inhibits the growth of endometriosis-like lesions and stromal fibrosis.

As for the endometriotic epithelial cells, we evaluated their expression of nuclear Ki67. We found that the ratio of Ki67-positive cells in the ASCs-P3-D1 and ASCs-P3-D15 groups was significantly lower than that 
in the Control-D28 and Control-D15 groups, respectively (Fig. 2D and Fig. 2E). These results suggest that endometriotic epithelial cells continue to proliferate in the endometriosis-like lesions, and that their proliferation is inhibited after infusion of ASCs with stemness potential.

\section{Alterations in target gene expression in endometriosis-like lesions mediated by the administration of ASCs}

To assess the molecular changes in endometriosis-like lesions induced by the administration of ASCs, we determined the mRNA expression levels of pro-and anti-inflammatory cytokines, pro-fibrotic cytokines, matrix metalloproteases, angiogenetic factors, and hormone receptors using quantitative RT-PCR. In the ASCs-P3-D1 and ASCs-P3-D1 groups, expression levels of monocyte chemotactic protein-1 (Mcp1), interleukin-6 (//6), and leukemia inhibitory factor (Lif) (pro-inflammatory cytokines) as well as tumor growth factor- $\beta 1$ (Tgfb1) (pro-fibrotic cytokine) were significantly lower than those in the Control-D28 and Control-D15 groups (Fig. 3). No significant differences in the expression levels of //10 and //4 (antiinflammatory cytokines), matrix metalloprotease-2 (Mmp2) and Mmp9 (matrix metalloproteases), vascular endothelial growth factor A (Vegfa) (angiogenetic factor), and estrogen receptor-1 (Er1) and progesterone receptor (Pgr) (hormone receptors) were found among the Control-D28, ASCs-P3-D1, Control-D15, and ASCs-P3-D15 groups (Fig. 3). These results suggest that pro-inflammatory and profibrotic cytokines are constantly expressed in endometriosis-like lesions, and that the administration of ASCs ameliorates endometriosis-like lesions by suppressing pro-inflammatory and pro-fibrotic cytokine expression.

\section{Tracking ASCs in endometriosis-like lesions in vivo}

In cell culture, Kusabira Orange (KuO)-ASCs originally displayed similar red/orange fluorescence as cells derived from KuO-expressing transgenic mice (Fig. 4A). Using phase-contrast and fluorescence microscopy, ASCs expressing KuO (KuO-ASCs), which were intravenously infused, appeared to accumulate in the endometriosis-like lesions (Fig. 4B). In the pathological specimens of endometriosislike lesions, KuO-ASCs displayed spindle and small fibroblast-like cell morphologies, and fluorescence microscopy revealed that their cytoplasm expressed a strong red/orange fluorescent signal and their nuclei were strongly stained with 4',6-diamidino-2-phenylindole (DAPI) (Fig. 4C). In addition, the intravenous administration of KuO-ASCs also revealed red/orange fluorescent signals in the pathological specimens of spleen and lungs (Supplementary Fig. S3). However, in other organs, including the brain, liver, kidney, heart, and peritoneum, no red/orange fluorescent signals were detected (Supplementary Fig. S3). These results suggest that intravenously infused ASCs predominantly accumulate in endometriosislike lesions but may systemically spread and finally accumulate in the spleen or lungs.

\section{Discussion}

In this study, the administration of ASCs was found to significantly inhibit the growth of endometriosislike lesions. In addition, ASCs displayed homing potential to integrate into endometriosis-like lesions, and 
suppressed the proliferation of endometriotic epithelial cells as well as the interstitial stromal reaction in a preclinical murine model of endometriosis. ASC infusion also decreased pro-inflammatory and profibrotic cytokine expression but did not alter the expression of anti-inflammatory cytokines, matrix metalloproteases, angiogenetic factors, or estrogen and progesterone receptors.

Endometrial stem cells, which are located in the basalis layer, differentiate into the endometrial stroma, epithelium, and endothelium [39]. After the infusion of bone-marrow cells with mTert-GFP or Ch $\beta$-actin GFP, all GFP-positive cells in the endometrium are immune cells, including $T$ cells and macrophages, suggesting that bone-marrow stem cells do not contribute to endometrial cell lineages [40]. In this study, infused ASCs were primarily detected in the endometriosis-like lesions, suggesting that they transdifferentiate into immune cells and help attenuate the stromal reaction in endometriosis-like lesions. Given that ASC administration significantly suppressed the thickness of the interstitial stroma and expression of pro-inflammatory and pro-fibrotic cytokines, it is plausible that ASCs, which were integrated into the institutional stromal tissues, suppress the expression of pro-inflammatory and pro-fibrotic cytokines that exacerbate the fibrotic changes in endometriosis. Indeed, ASC treatment significantly reduced the total number as well as the total weight and surface area of endometriosis-like lesions.

II-6 levels are elevated in the peritoneal fluid, endometriotic lesions, and serum from women with endometriosis [41, 42], and TGF- $\beta 1$ is also upregulated in the peritoneal fluid and peritoneal membranes of women with endometriosis $[43,44]$. In other preclinical models of endometriosis, it has been reported that the increase in IL- 6 and TGF- $\beta 1$ levels is attributable to the development of endometriosis-like lesions $[13,14]$. MSCs, which retain immunomodulatory features and secrete trophic factors, have been utilized for treating various inflammatory and fibrotic diseases [45]. Several studies have demonstrated that MSC infusion attenuates fibrosis through the suppression of IL-6 or TGF- $\beta 1$ expression [46, 47]. In this study, the administration of ASCs also reduced the expression of IL-6, MCP-1, Lif- 1 , and TGF- $\beta 1$, accompanied by the attenuation of endometriosis-like lesions. Although the immunomodulatory and trophic factors remain to be unidentified [48], MCS infusion may represent a promising treatment for endometriosis.

Although MSCs have been previously shown to suppress the function of anti-inflammatory and angiogenic cytokines [48], such findings were not detected in this study. Here, the proliferation ratio of endometriotic epithelial cells in the treated groups was significantly suppressed and no clear differences in VEGF, MMP2, MMP9, IL-4, and IL-10 expression was found between the treated and control groups. In another experimental murine model of endometriosis, the infusion of anti-IL- 6 antibody induced ectopic endometriotic epithelial cell atrophy [49]. Although it remains unclear why the growth of endometriotic epithelial cells was inhibited by ASC treatment, the suppression of IL-6 expression, which was mediated by ASC infusion, may have resulted in the decreased proliferation of endometriotic epithelial cells.

The safety and efficacy of infused MSCs have been validated by clinical trials in patients with a variety of diseases [45]. Moreover, ASC treatment had no influence on the expression of estrogen and progesterone receptors, suggesting that combination therapy involving hormonal therapy and MSC infusion would synergistically act to significantly attenuate endometriosis. Many women with endometriosis continue to 
suffer from severe dysmenorrhea, infertility, cancer, and other debilitating conditions. To overcome the incurable disorders associated with endometriosis, clinical trials investigating the potential of MSCs should be initiated for patients with endometriosis to preserve ovarian function and fertility.

\section{Methods}

\section{Animals}

Six-week-old BALB/c female mice were obtained from KBT Oriental (Saga, Japan). A KuO-expressing transgenic C57BL/ 6 mouse was provided by Dr. H. Nakauchi at the Institute of Medical Science, University of Tokyo [34]. All experiments were performed in accordance with the Guidelines for Animal Experiments at Fukuoka University and approved by the Animal Experiment Committee of Fukuoka University (approval number: 1806020, 2005007) and the Safety Committee for Recombinant DNA Experiments of Fukuoka University (approval number: 563). The study was carried out in compliance with the ARRIVE guidelines.

\section{Isolation of adipose-derived regenerative cells (ADRCs) and ASCs}

We isolated ADRCs from the subcutaneous adipose tissues of BALB/c female mice and the KuOexpressing transgenic C57BL/6 mouse, as described previously [35]. ADRCs are almost identical to the stromal vascular fraction. To obtain ACSs, ADRCs were incubated on a $100 \mathrm{~mm}$ plate in $10 \mathrm{~mL}$ of MEMa/GlutaMax medium (Gibco, Grand Island, NY, USA) containing 20\% fetal bovine serum (Gibco) and $1 \%$ antibiotic-antimycotic ( $\mathrm{Gibco}$ ) at $37^{\circ} \mathrm{C}$ and $5 \% \mathrm{CO}_{2}$ until $80 \%$ confluence. The medium was replaced every 3 days to remove nonadherent cells, and the adherent cells, which were considered ASCs, were passaged with TrypLE Express (Gibco). ASCs obtained at P3 and P20 were utilized, and cell numbers were counted using Luna-FL (Logos Biosystems, Anyang-si, South Korea). To determine the percentage of cells with stemness potential in P3 and P20 ACSs, the characteristics of ACSs were independently examined using flow cytometric analysis in triplicate.

\section{Animal experiments}

The endometriosis murine model was established as previously described [36] based on the retrograde shed endometrium theory. The experimental murine model of endometriosis is presented in Supplementary Fig. S1. Donor and recipient mice, which were ovariectomized after acclimation, were subcutaneously administered $100 \mu \mathrm{g} / \mathrm{kg}$ estradiol valerate (Tokyo Chemical Industry Co., Ltd, Tokyo, Japan) twice (day - 14 and - 7) for 2 weeks immediately after ovariectomy. Donor mice were sacrificed, and then their uterine horns were removed and minced as uterine fragment tissues. Half of the uterine fragment tissues obtained per mouse were suspended in $2 \mathrm{~mL}$ of PBS and injected into the peritoneal cavity of recipient mice with an 18-gauge needle (day 0 ). On day 28 , these recipient mice were sacrificed, and the endometriosis-like lesions excised. Mice intravenously administered PBS $(0.5 \mathrm{~mL})$ on day 1 , instead of receiving the ASCs infusion, were defined as control (Control-D28 group). Mice that were 
intravenously administered $1 \times 10^{6}$ P3 ASCs and $1 \times 10^{6}$ P20 ASCs in PBS $(0.5 \mathrm{~mL})$ on day 1 and sacrificed on day 28 were defined as the ASCs-P3-D1 and ASCs-P20-D1 groups, respectively; before administration, the ASCs were suspended in $0.5 \mathrm{~mL}$ of PBS.

To confirm the anti-tumor effects of ASCs on endometriosis-like lesions that had already formed, recipient mice that had not received any treatment were sacrificed and endometriosis-like lesions were collected on day 15 after the injection of uterine fragment tissues on day 0 (Control-D15 group). In addition, some mice were intravenously treated with $1 \times 10^{6} \mathrm{P} 3$ ASCs and $1 \times 10^{6}$ P20 ASCs in PBS $(0.5 \mathrm{~mL})$ and sacrificed on day 28, after which endometriosis-like lesions were collected; these mice were defined as the ASCs-P3-D15 and ASCs-P20-D15 groups, respectively. For administration, $1 \times 10^{6}$ ASCs in PBS or PBS only were injected into the tail vein of mice using a 25-gauge needle on day 1 or day 15 .

\section{Flow cytometric, pathological, and immunohistochemical analyses}

ASCs were suspended in a staining buffer (R\&D Systems, Minneapolis, MN, USA), followed by incubation with a mouse primary antibody against the Fc component for $10 \mathrm{~min}$ at $4^{\circ} \mathrm{C}$ to block nonspecific binding. They were then incubated for $30 \mathrm{~min}$ at $25^{\circ} \mathrm{C}$ with CD105-FITC, CD29-PE, Sca-1-APC, and CD45-PerCP (all from the Mouse Mesenchymal Stem Cell [MSC] Multi-Color Flow Kit, cat. no. FMC003; R\&D Systems). Flow cytometry was performed as described previously [35]. For histological staining, the collected tissues were formalin-fixed and then paraffin-embedded. Next, the tissue samples were sliced into 4- $\mu m$ sections and stained with hematoxylin and eosin. Stromal thickness was evaluated by comparing the mean maximum width of each lesion. To evaluate fibrosis in endometriosis-like lesions, Masson's trichrome staining was performed using pathological specimens of the lesions. To assess the proliferative ratio of endometriotic epithelial cells in endometriosis-like lesions, the primary antibody against Ki67 (1:200; Proteintech) was used. In a single section of each lesion, the Ki67-positive cell ratio was calculated by counting Ki67-positive epithelial cell nuclei per total epithelial cell nuclei. The pathological parameters, including the mean maximum width of the stromal tissues and proliferative ratio of endometriotic epithelial cells in endometriosis-like lesions, were independently estimated by three examiners (K.K., M.A., and K.N), and the mean value was defined as a representative value for each sample.

\section{Quantitative reverse transcription PCR}

Collected tissues were incubated in RNAlater reagent (Thermo Fisher Scientific, Waltham, MA, USA) at $4^{\circ} \mathrm{C}$ overnight. Total RNA was extracted from tissues using the RiboPure RNA Purification Kit (Thermo Fisher Scientific), and the concentration and purity were estimated by measuring the $A_{260} / A_{280}$ ratio (where " $A$ " denotes absorbance) on the NanoDrop 2000 spectrophotometer (Thermo Fisher Scientific). cDNA was generated by reverse transcription of the extracted RNA $(1 \mu \mathrm{g})$ using the PrimeScript II 1st Strand cDNA Synthesis Kit (TaKaRa Bio Inc., Shiga, Japan). The mRNA levels were quantified using the StepOne realtime PCR system (Applied Biosystems, Waltham, MA, USA). TaqMan probes specific for Mcp1, I/6, Lif, Tgfb1, II4, I/10, Vegfa, Mmp2, Mmp9, Er1, Pgr, and glyceraldehyde-3-phosphate dehydrogenase (Gapdh) 
were used for real-time PCR. The absolute values of Mcp1, Il6, Lif, Tgfb1, I/4, I/10, Vegfa, Mmp2, Mmp9, Er1, and Pgrwere normalized to that of Gapdh, and the relative values were calculated by comparison with the control group. All samples were examined in triplicate.

\section{Tracking transplanted ASCs expressing KuO}

KuO-ASCs were isolated from a KuO-expressing transgenic mouse. Approximately $1 \times 10^{6} \mathrm{KuO}-\mathrm{ASC}$ were injected either intraperitoneally or intravenously into the endometriosis model mice on day 1 . To track the distribution of the infused KuO-ASCs in mice with endometriosis, a variety of organs, including the peritoneum, liver, spleen, kidneys, lungs, heart, and brain, were excised and prepared as frozen sections at $24 \mathrm{~h}$ post-KuO-ASC infusion as previously described [37, 38]. The tissue sections were fixed, washed, and incubated with DAPI. All sections were examined using a fluorescence microscope (Keyence, Osaka, Japan). Control mice were intravenously injected with $0.5 \mathrm{~mL}$ of PBS on day 1 and sacrificed on day 2 (Control-D2).

\section{Statistical analysis}

All data were evaluated using the Prism 8 software (GraphPad Software Inc., San Diego, CA, USA). The Mann-Whitney $\mathrm{U}$ test was performed to compare the total weight, number, and surface area of the lesions; stromal thickness; percentage of Ki67-positive cells; and expression levels of target genes determined via quantitative RT-PCR among different groups. The data are presented as the mean \pm standard error of the mean. $P<0.05$ was considered statistically significant.

\section{Declarations}

\section{Data availability}

The datasets generated during and/or analyzed during the current study are available from the corresponding author on reasonable request.

\section{Acknowledgements}

We appreciate the excellent technical assistance from Ms. M. Onitsuka, Department of Pathology, Fukuoka University School of Medicine and Hospital. This work was supported in part by JSPS KAKENHI (grant number JP18K09242); the Central Research Institute of Fukuoka University (grant number 197011); a Grant-in-Aid from the Kakihara Science and Technology Foundation (Fukuoka, Japan) to S. Miyamoto (grant number 180381) and K. Miyata (grant number 190412); and a Grant-in-Aid from the Society for Women's Health Science Research (Osaka, Japan) to S. Miyamoto (grant number 190064). The funding sources played no role in the study design; in the collection, analysis, and interpretation of data; in the writing of the report; or the decision to submit the article for publication. We thank Editage (https://www.editage.jp/) for English Language editing.

\section{Author contributions}


S.M., F.Y., and T.H. initiated and designed the study. K.K., Y.O., H.K., F.T., and T.H. supervised the preparation of the experimental murine model of endometriosis. K.K., M.A., and K.N. performed the pathological analysis including hematoxylin and eosin, Masson's trichrome, and immunohistochemical staining. N.S. and S.Y. supervised and performed flow cytometry. C.K., D.U., and K.Y. performed adiposederived stem cell extraction and subculturing. K.M. and M.K. performed real-time PCR. T.H. contributed to all experiments. S.M. conceived of the study was involved in planning and supervising the work. T.H. and S.M. wrote and edited the manuscript. All authors discussed the results and commented on the manuscript.

\section{Competing interests}

The authors declare no competing interests.

\section{References}

1. Clement, P. B. The pathology of endometriosis: a survey of the many faces of a common disease emphasizing diagnostic pitfalls and unusual and newly appreciated aspects. Adv. Anat. Pathol. 14, 241-260 (2007). doi: 10.1097/PAP.0b013e3180ca7d7b.

2. Giudice, L. C. Endometriosis. N. Engl. J. Med. 362, 2389-2398 (2010). doi:

10.1056/NEJMcp1000274.

3. D'Hooghe, T. M. Endometriosis in Berek \& Novak's Gynecology (15th Ed.) (eds. Berek, J. S. \& Novak, E) 505-556 (Lippincott Williams \& Wilkins, 2011).

4. Hickey, M., Ballard, K. \& Farquhar, C. Endometriosis. BMJ. 348, g1752 (2014). doi: 10.1136/bmj.g1752.

5. Aris, A. Endometriosis-associated ovarian cancer: a ten-year cohort study of women living in the Estrie Region of Quebec, Canada. J. Ovarian Res. 3, 2 (2010). doi: 10.1186/1757-2215-3-2.

6. Suryawanshi, S., et al. Complement pathway is frequently altered in endometriosis and endometriosis-associated ovarian cancer. Clin. Cancer Res. 20,6163-6174 (2014). doi: 10.1158/1078-0432.CCR-14-1338.

7. Vercellini, P., Viganò, P., Somigliana, E. \& Fedele, L. Endometriosis: pathogenesis and treatment. Nat. Rev. Endocrinol. 10, 261-275 (2014).

8. Viganò, P., et al. Cellular components contributing to fibrosis in endometriosis: A literature review. J. Minim. Invasive Gynecol. 27, 287-295 (2020).

9. Strippoli, R., et al. Molecular mechanisms underlying peritoneal EMT and fibrosis. Stem Cells Int. 2016, 3543678 (2016). doi: 10.1155/2016/3543678

10. Jin, X., Ren, S., Macarak, E. \& Rosenbloom J. Pathobiological mechanisms of peritoneal adhesions: The mesenchymal transition of rat peritoneal mesothelial cells induced by TGF- $\beta 1$ and IL- 6 requires activation of Erk1/2 and Smad2 linker region phosphorylation. Matrix Biol. 51, 55-64 (2016). 
11. Falk, P., Angenete, E., Bergström, M. \& Ivarsson, M. L. TGF- $\beta 1$ promotes transition of mesothelial cells into fibroblast phenotype in response to peritoneal injury in a cell culture model. Int. J. Surg. 11, 977982 (2013).

12. Agha, E. E., et al. Mesenchymal stem cells in fibrotic disease. Cell Stem Cell. 21, 166-177 (2017). doi: 10.1016/j.stem.2017.07.011.

13. Song, Y., et al. Interleukin-6 (IL-6) activates the NOTCH1 signaling pathway through E-proteins in endometriotic lesions. J. Clin. Endocrinol. Metab. 105, 1316-1326 (2020).

14. Soni, U. K., et al. A high level of TGF-B1 promotes endometriosis development via cell migration, adhesiveness, colonization, and invasiveness. Biol. Reprod. 100, 917-938 (2019).

15. Fuchs, E. \& Segre, J. A. Stem cells: a new lease on life. Cell 100, 143-155 (2000). doi: 10.1016/S0092-8674(00)81691-8.

16. Zuk, P. A., et al. Multilineage cells from human adipose tissue: implications for cell-based therapies. Tissue Eng. 7, 211-228 (2001). doi: 10.1089/107632701300062859.

17. Lerou, P. H. \& Daley G. Q. Therapeutic potential of embryonic stem cells. Blood Rev. 19, 321-331 (2005). doi: 10.1016/j.blre.2005.01.005.

18. Kolios, G. \& Moodley Y. Introduction to stem cells and regenerative medicine. Respiration 85, 3-10 (2013). doi: 10.1159/000345615.

19. Wei, X., et al. Mesenchymal stem cells: a new trend for cell therapy. Acta Pharmacol. Sin. 34, 747754 (2013). doi: 10.1038/aps.2013.50.

20. Bacakova, L., et al. Stem cells: their source, potency and use in regenerative therapies with focus on adipose-derived stem cells - a review. Biotechnol. Adv. 36, 1111-1126 (2018). doi: 10.1016/j.biotechadv.2018.03.011.

21. Ptaszek, L. M., Mansour, M., Ruskin, J. N. \& Chien, K. R. Towards regenerative therapy for cardiac disease. Lancet 379, 933-942 (2012). doi: 10.1016/S0140-6736(12)60075-0.

22. Li, M., Ma, J., Gao, Y. \& Yang, L. Cell sheet technology: a promising strategy in regenerative medicine. Cytotherapy 21, 3-16 (2019). doi: 10.1016/j.jcyt.2018. 10.013.

23. Weinberg, R. S. Overview of cellular therapy in Transfusion medicine and homeostasis (eds. Shaz, B.H., Hillyer, C.D. \& Gil, M.R.) 505-512 (Elsevier, 2019).

24. Musiał-Wysocka, A., Kot, M. \& Majka, M. The pros and cons of mesenchymal stem cell-based therapies. Cell Transplant. 28, 801-812 (2019). doi: 10.1177/ 0963689719837897.

25. Bhartiya, D. \& Mohammad, S. A. Which stem cells will eventually translate to the clinics for treatment of diabetes? Stem Cell Res. Ther. 11, 211 (2020). doi: 10.1186/s13287-020-01718-3.

26. Andrzejewska, A., Lukomska, B., \& Janowski, M. Concise Review: Mesenchymal stem cells: From roots to boost. Stem Cells 37, 855-864 (2019).

27. Zhao, Y. \& Zhang H. Update on the mechanisms of homing of adipose tissue-derived stem cells. Cytotherapy 18, 816-827 (2016). doi: 10.1016/j.jcyt. 2016.04.008. 
28. Sarveazad, A., et al. Comparison of human adipose-derived stem cells and chondroitinase ABC transplantation on locomotor recovery in the contusion model of spinal cord injury in rats. Iran J. Basic Med. Sci. 17, 685-693 (2014).

29. Menezes, K., et al. Human mesenchymal cells from adipose tissue deposit laminin and promote regeneration of injured spinal cord in rats. PLoS One. 9, e96020 (2014). doi:

10.1371/journal.pone.0096020.

30. Haubner, F., et al. A co-culture model of fibroblasts and adipose tissue-derived stem cells reveals new insights into impaired wound healing after radiotherapy. Int. J. Mol. Sci. 16, 25947-25958 (2015). doi: 10.3390/ijms161125935.

31. Cho, Y. B., et al. Long-term results of adipose-derived stem cell therapy for the treatment of Crohn's fistula. Stem Cells Transl. Med. 4, 532-537 (2015). doi: 10.5966/sctm.2014-0199.

32. Ammar, H. I., et al. Comparison of adipose tissue- and bone marrow-derived mesenchymal stem cells for alleviating doxorubicin-induced cardiac dysfunction in diabetic rats. Stem Cell Res. Ther. 6, 148 (2015). doi: 10.1186/s13287-015-0142-x.

33. Lee, H. W., et al. Effects of intracoronary administration of autologous adipose tissue-derived stem cells on acute myocardial infarction in a porcine model. Yonsei Med. J. 56, 1522-1529 (2015). doi: 10.3349/ymj.2015.56.6.1522.

34. Hamanaka, S., et al. Generation of transgenic mouse line expressing Kusabira Orange throughout body, including erythrocytes, by random segregation of provirus method. Biochem. Biophys. Res. Commun. 435, 586-591 (2013). doi: 10.1016/j.bbrc. 2013.05.017.

35. Yotsumoto, F., et al. Adipose tissue-derived regenerative cells improve implantation of fertilized eggs in thin endometrium. Regen. Med. 15, 1891-1904 (2020). doi: 10.2217/rme-2020-0037.

36. Takamura, M., et al. Neutrophil depletion reduces endometriotic lesion formation in mice. Am. J. Reprod. Immunol. 76, 193-198 (2016). doi: 10.1111/aji.12540.

37. Watanabe, Y., et al. Mesenchymal stem cells and induced bone marrow-derived macrophages synergistically improve liver fibrosis in mice. Stem Cells Transl. Med. 8, 271-284 (2019).

38. Ling, L., et al. Human amnion-derived mesenchymal stem cell (hAD-MSC) transplantation improves ovarian function in rats with premature ovarian insufficiency (POI) at least partly through a paracrine mechanism. Stem Cell Res. Ther. 10, 46 (2019). doi: 10.1186/s13287-019-1136-x.

39. Cousins, F. L., Pandoy, R., Jin, S. \& Gargett, C. E. the elusive endometrial epithelial stem/progenitor cells. Front. Cell Dev. Biol. 9, 640319 (2021). doi: 10.3389/fcell.2021.640319.

40. Ong, Y. R., et al. Bone marrow stem cells do not contribute to endometrial cell lineages in chimeric mouse models. Stem Cells. 36, 91-102 (2018).

41. Volpato, L. K., Horewicz, V. V., Bobinski, F., Martins, D. F. \& Piovezan, A. P. Annexin A1, FPR2/ALX, and inflammatory cytokine expression in peritoneal endometriosis. J. Reprod. Immunol. 129, 30-35 (2018).

42. Jaeger-Lansky, A., et al. Local and systemic levels of cytokines and danger signals in endometriosisaffected women. J. Reprod. Immunol. 130, 7-10 (2018). 
43. Young, V. J., et al. Transforming growth factor- $\beta$ induced Warburg-like metabolic reprogramming may underpin the development of peritoneal endometriosis. J. Clin. Endocrinol. Metab. 99, 3450-3459 (2014).

44. Young, V. J., Ahmad, S. F., Brown, J. K., Duncan, W. C. \& Horne, A. W. Peritoneal VEGF-A expression is regulated by TGF- $\beta 1$ through an ID1 pathway in women with endometriosis. Sci. Rep. 5, 16859 (2015).

45. Ryu, J. S., et al. Application of mesenchymal stem cells in inflammatory and fibrotic diseases. Int. J. Mol. Sci. 21, 8366 (2020). doi: 10.3390/ijms21218366.

46. Luo, X. Y., et al. Transplantation of bone marrow mesenchymal stromal cells attenuates liver fibrosis in mice by regulating macrophage subtypes. Stem Cell Res. Ther. 10, 16 (2019). doi: 10.1186/s13287-018-1122-8.

47. Liao, Z., Liu, C., Wang, L., Sui, C. \& Zhang, H. therapeutic role of mesenchymal stem cell-derived extracellular vesicles in female reproductive diseases. Front. Endocrinol. (Lausanne). 12, 665645 (2021). doi: 10.3389/fendo.2021.665645.

48. Brigstock, D. R. Extracellular vesicles in organ fibrosis: Mechanisms, therapies, and diagnostics. Cells. 10, 1596 (2021). doi: 10.3390/cells10071596.

49. El-Zayadi, A. A., et al. Anti-IL-6 receptor monoclonal antibody as a new treatment of endometriosis. Immunol. Res. 68, 389-397 (2020).

\section{Figures}


A
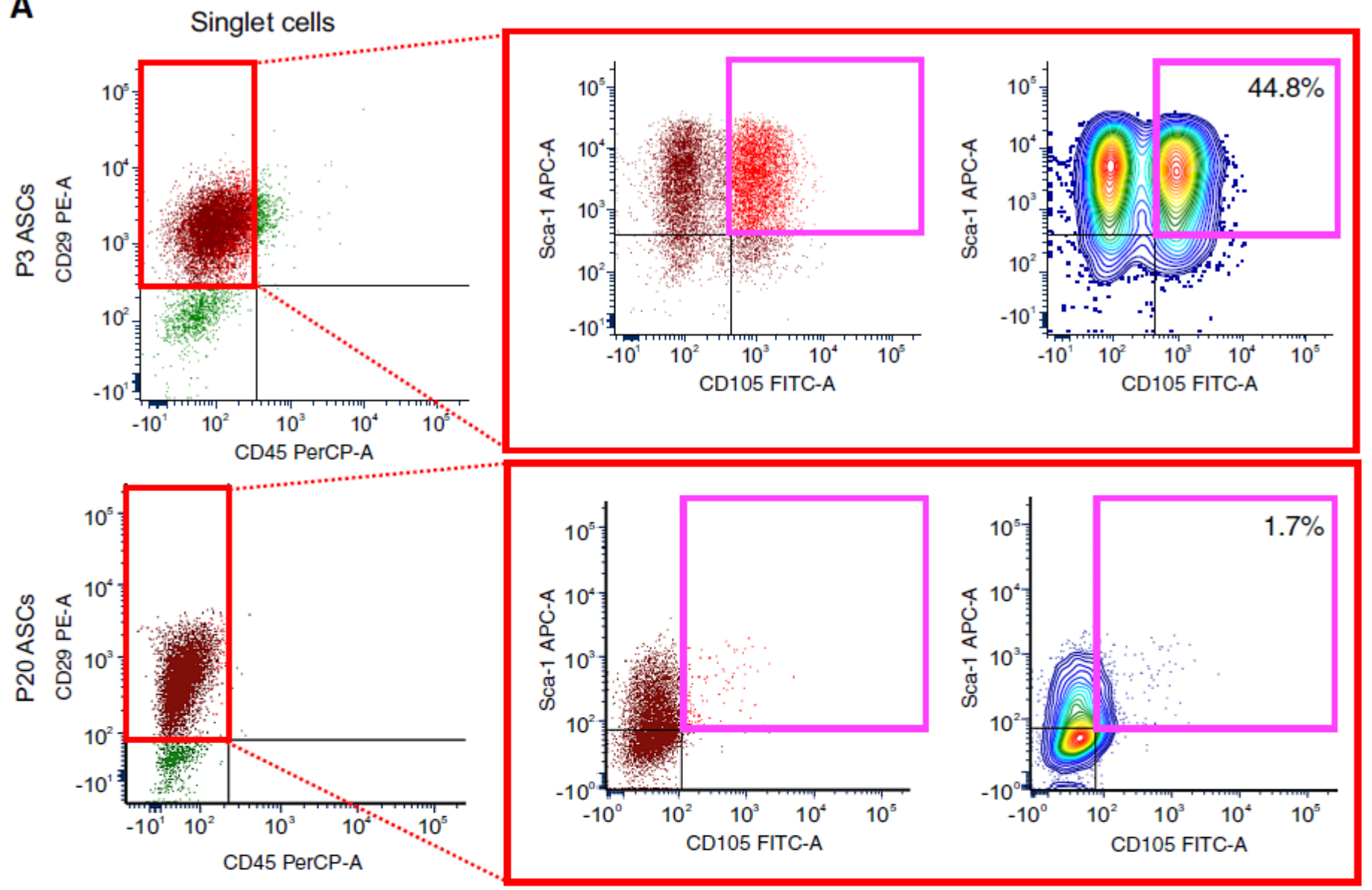

B
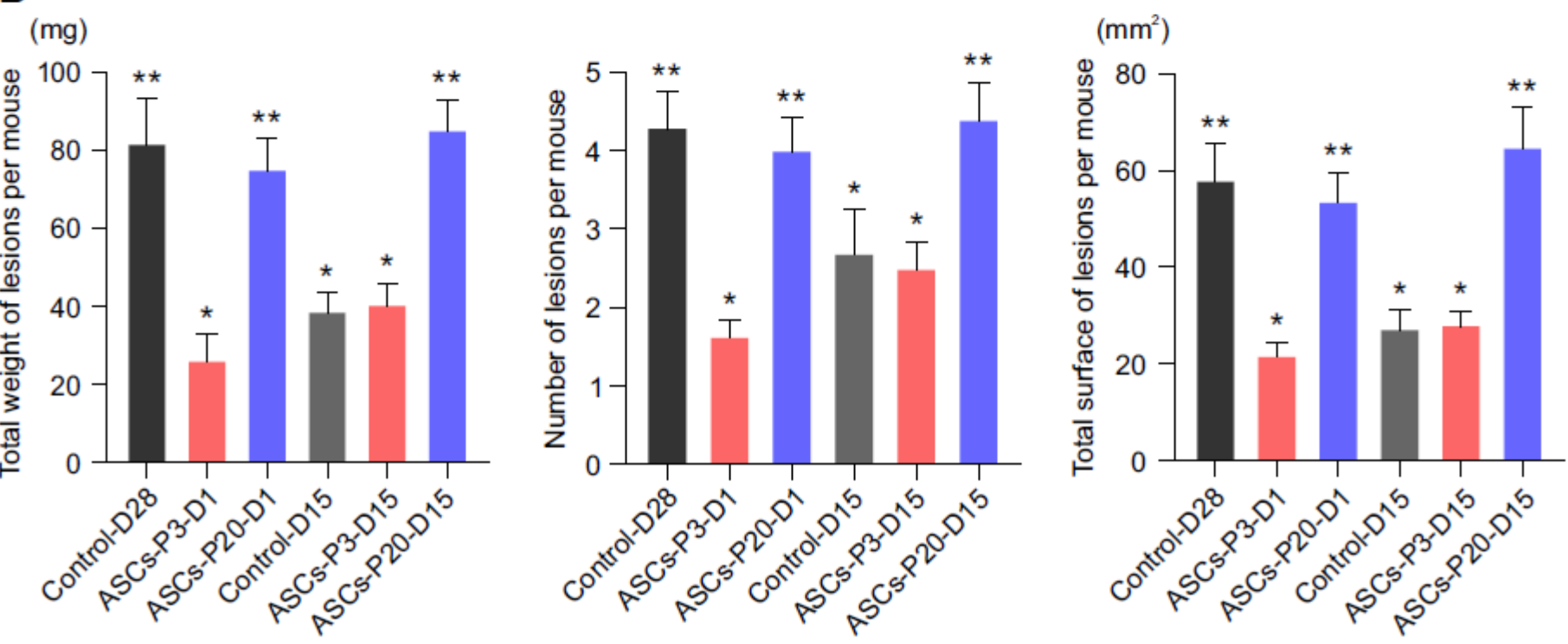

Figure 1

ASC stemness potential in endometriosis-like lesions. (A) Multicolor flow cytometry analysis of cells in the plot of singlet cells based on staining for CD105, CD29, Sca-1, and CD45. The percentages of mesenchymal stem cells, including third passage (P3) adipose tissue-derived stem cells (ASCs), and P20 ASCs, harboring the cell-surface expression markers CD29, Sca-1, and CD105, and negative CD45 are shown. (B) Changes in secondary morphology of endometriosis-like lesion configuration after treatment 
with ASCs. Control-D28: intravenous administration of only PBS on day 1; ASCs-P3-D1: intravenous administration of P3 ASCs on day 1; ASCs-P20-D1: intravenous administration of P20 ASCs on day 1; Control-D15: sacrificed on day 15; ASCs-P3-D15: intravenous administration of P3 ASCs on day 15; and ASCs-P20-D15: intravenous administration of P20 ASCs on day 15. Except for Control-D15 group, the mice were sacrificed on day 28. All groups included 10 mice. Data are presented as the mean \pm standard error. ${ }^{*} \mathrm{P}<0.05 ;{ }^{*} \mathrm{P}<0.05$ vs. Control-D28 or ASCs-P3-D1.

A

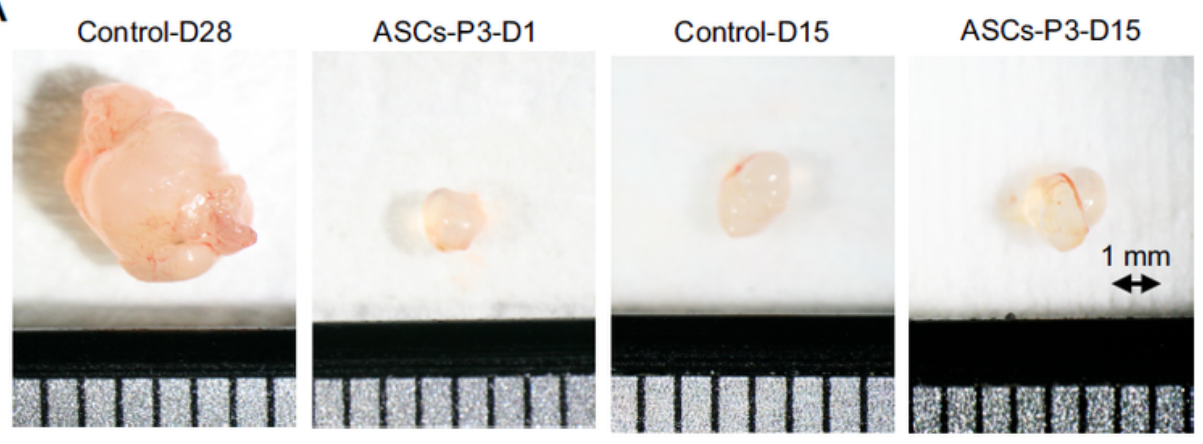

B

Control-D28

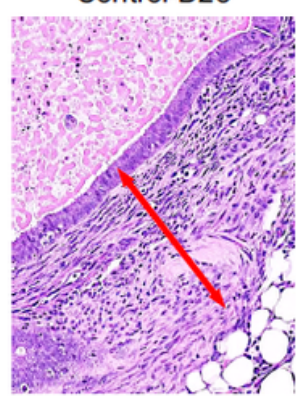

C

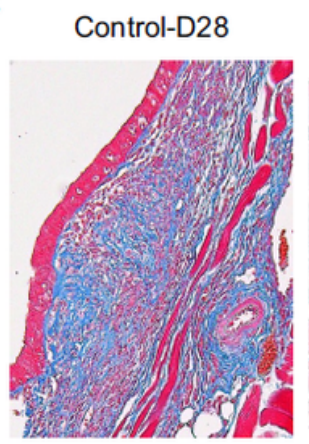

D

Control-D28

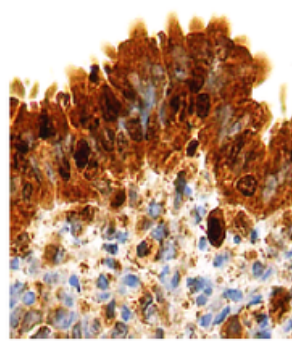

ASCs-P3-D1

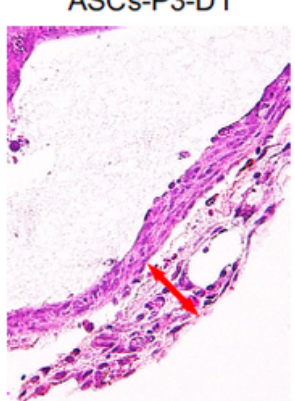

ASCs-P3-D1

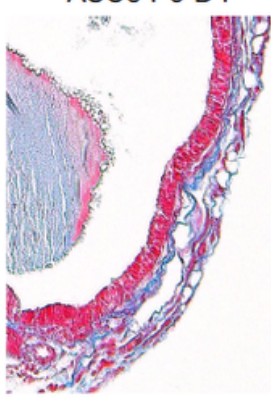

ASCs-P3-D1

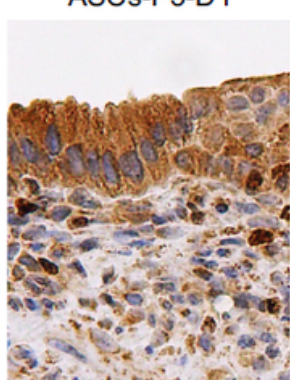

Control-D15
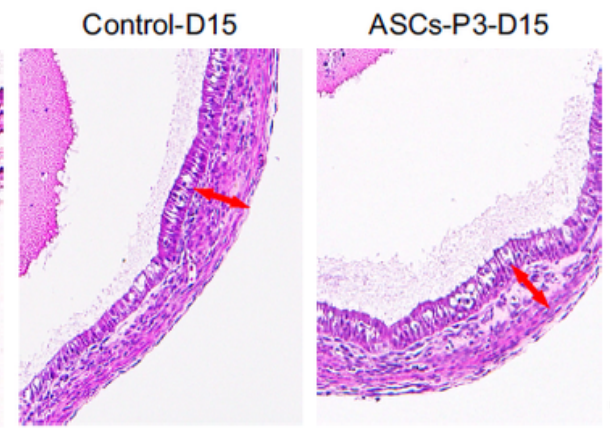

$100 \mu \mathrm{m}$
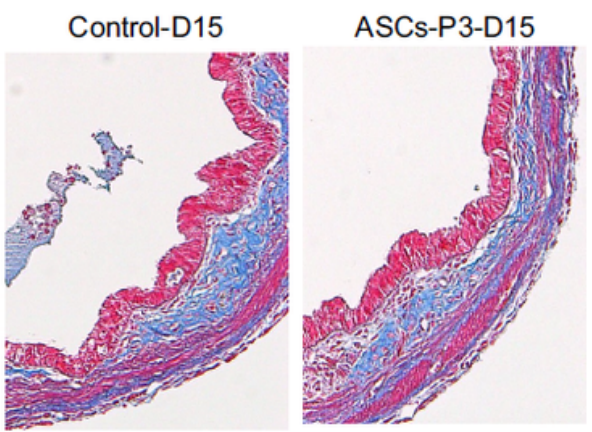

Control-D15

ASCs-P3-D15

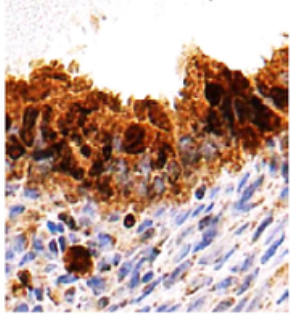

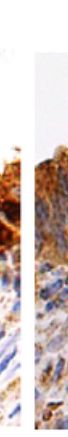

E

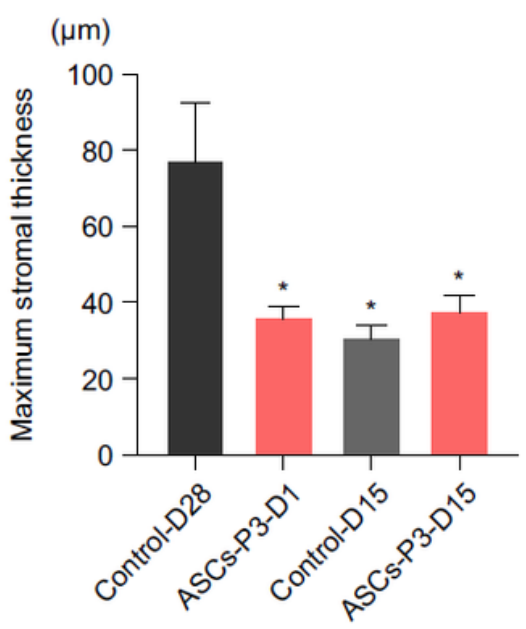

(\%)

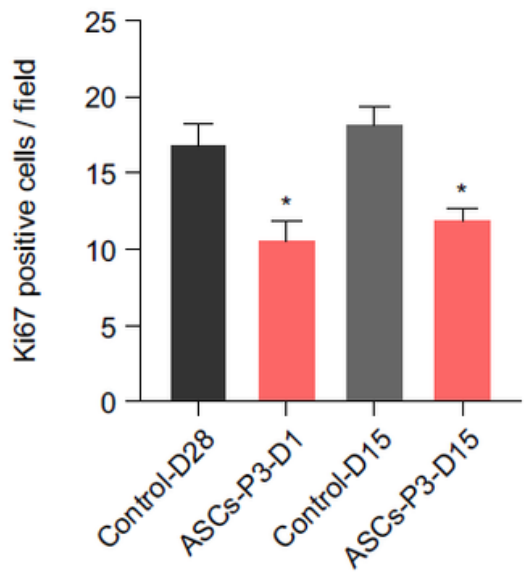

Figure 2 
Macroscopic alterations and histopathology of endometriosis-like lesions after treatment with ASCs. (A) Representative excised implants from the endometriosis-like lesions. The interval at the bottom represents $1 \mathrm{~mm}$. (B-D) Representative images of pathological specimens from excised implants stained with hematoxylin and eosin (B), Masson's trichrome (C), and Ki67 (D). The red arrow in (B) indicates the maximum stromal thickness from the bottom of the endometrium to the surface of each cyst. The blue and red stains in (C) indicate collagen fibers and cells (mainly: fibroblasts) in the endometriosis-like lesions. (E) Quantification of maximum stromal thickness from the bottom of the endometrium to the surface of each cyst (top) and the percentage of Ki67-positive cells stained in the endometriotic epithelial cells (bottom). Data are presented as the mean \pm standard error. ${ }^{*} \mathrm{P}<0.01$ vs. Control-D28.
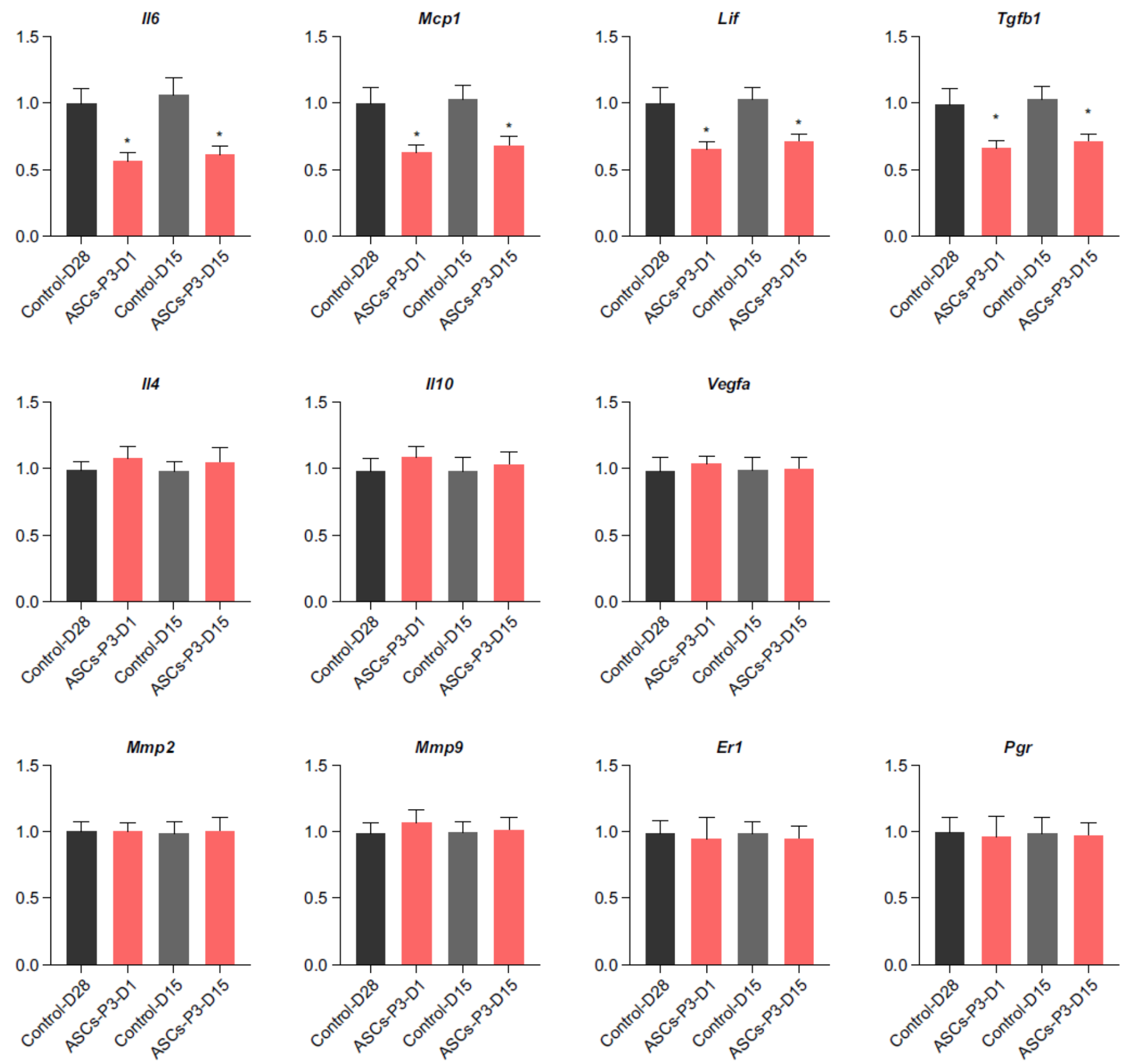

Figure 3 
Changes in gene expression in endometriosis-like lesions after infusion of ASCs. Quantitative RT-PCR of pro-inflammatory (II6, Mcp1,Lif), pro-fibrotic (Tgfb1), and anti-inflammatory cytokine (II4, I110) as well as angiogenetic factor (Vegfa), matrix metalloprotease (Mmp2, Mmp9), and hormone receptor (Er1, Pgr) gene expression levels in endometriosis-like lesions. Each group included 10 mice. Data are presented as the mean \pm standard error. ${ }^{*} \mathrm{P}<0.05$ vs. Control-D28.
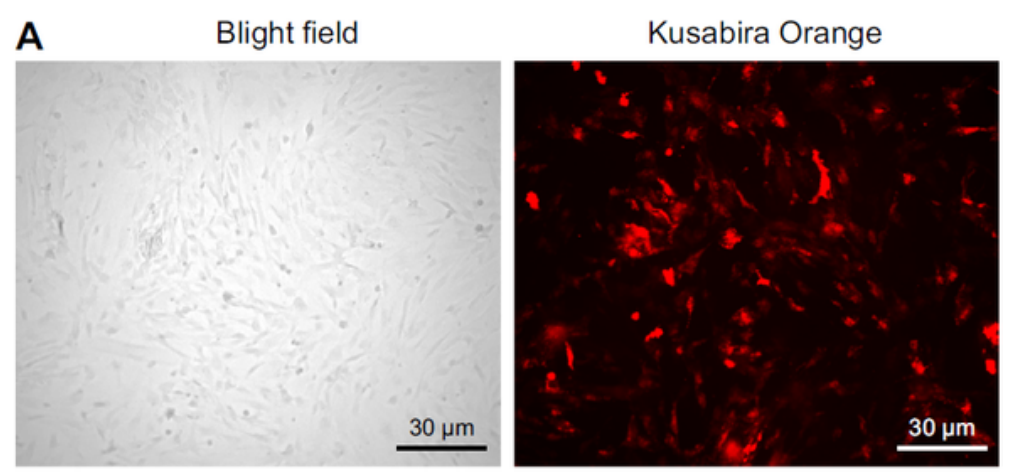

B

Blight field

Kusabira Orange
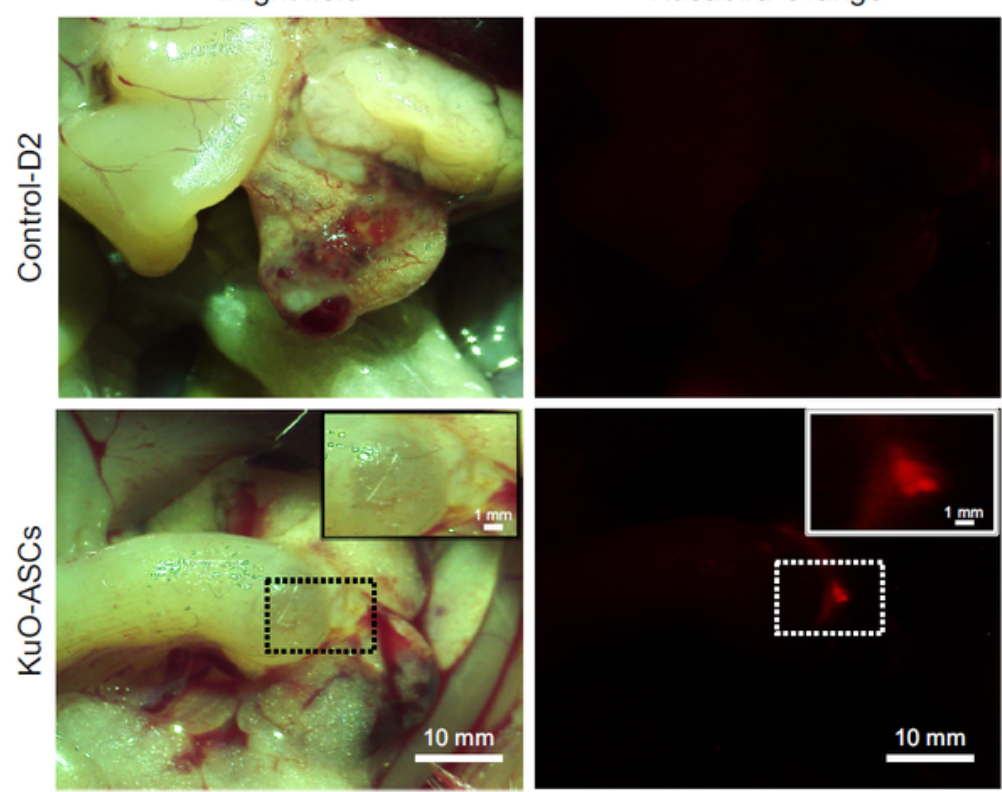

C

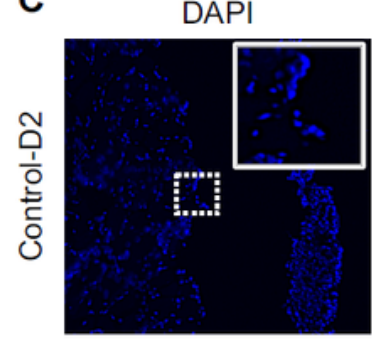

Kusabira Orange

Overlay
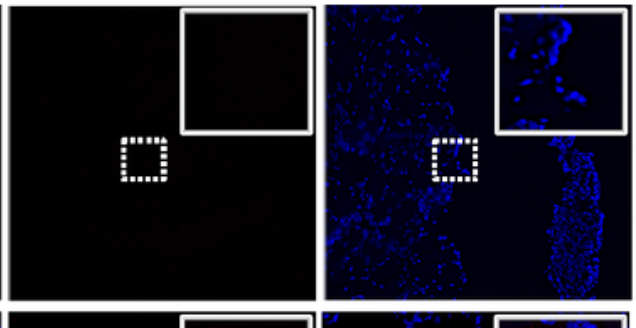

0
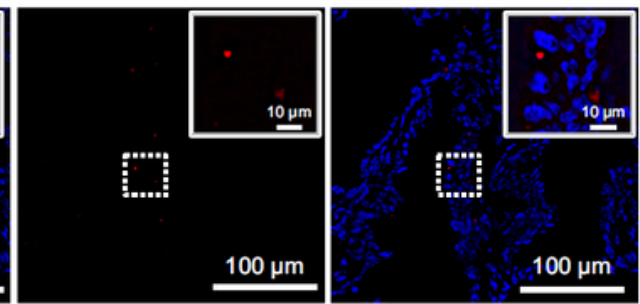

Figure 4 
Localization of KuO-ASCs after intravenous administration. (A-C) Phase-contrast and fluorescence microscopy showing the cell morphology of Kusabira Orange (KuO)-expressing ASCs (A); macroscopic localization of KuO-ASCs in the endometriosis-like lesions (B); and microscopic localization of KuO-ASCs in the endometriosis-like lesions (C). Nuclei were counterstained with 4',6-diamidino-2-phenylindole (DAPI).

\section{Supplementary Files}

This is a list of supplementary files associated with this preprint. Click to download.

- SupplementaryInformation.docx 